\title{
UN PENDIENTE HELENISTICO DE LAS EXCAVACIONES DEL ANFITEATRO DE CARTAGENA
}

\author{
José PÉREZ BALLESTER
}

El ejemplar que presentamos para su estudio fue hallado junto al Anfiteatro de Cartagena durante las excavaciones de 1968, y actualmente se halla expuesto en el Museo Arqueológico Municipal de esa ciudad. Se trata de un pendiente de oro en forma de disco, del que penden cinco colgantes: una anforita y cuatro cadenillas (fig. 1).

El disco, con un diámetro de 1,4 cm., presenta un pequeño granate circular engastado en su centro. Alrededor, y concéntricos a la piedra, un hilo de otro trenzado y otro más grueso, segmentado. El disco está circundado por una cenefa formada por triángulos o "dientes de lobo" cubiertos de un fino granulado.

El colgante principal es una anforita de peana cuadrada, cuerpo fusiforme, cuello y boca esbeltamente trabajados, y dos grandes asas que sobrepasan ampliamente el borde del ánfora, donde apoyan describiendo dos grandes volutas; están adornadas con granulado, al igual que el cuerpo, cubierto por triángulos y rombos granulados, que apenas dejan alguna superficie lisa. Su tamaño es de $1,9 \mathrm{~cm}$.

Los colgantes laterales son todos idénticos, y constan de unas cadenillas trenzadas de cuatro cabos, acabadas en campánulas o florecillas. Tienen una longitud que oscila entre 2 y $2,4 \mathrm{~cm}$.

Un grueso hilo de oro doblado engancha el ánfora al disco, sirviendo igualmente para su engarce en la oreja.

Aunque la configuración general de la joyita es clásica (disco principal del que cuelga una figura central y dos o cuatro colgantes secundarios), es sabido que este tipo de pendientes continúa elaborándose durante todo el período helenístico, dándose incluso en la orfebrería romana.

Es la presencia de una incipiente policromía (rojo del granate frente al oro) que no altera sin embargo el concepto de "joyita de oro" que define al pendiente, la que hace más fácil su situación dentro de la amplia época helenística: la utilización de piedras preciosas, perlas, cristal de roca, esmaltes, en la elaboración de joyitas de oro, sólo tendrá lugar después de las conquistas de Alejandro (a partir del 330 a. C.), cuando se abren definitivamente a Occidente las vías hacia los metales preciosos y también las modas y usos orientales de Persia, Asia Menor, Egipto, etc. 
Precisamente será el granate la piedra más comúnmente utilizada en el primer período del helenismo, entre el 330 y el siglo II a. C. junto a otras piedras o materiales de color rojo, como cornalinas, calcedonias, etc. (1).

El minucioso trabajo de filigrana de oro y el uso casi exclusivo del granulado, completan la elaboración de nuestra pieza.

Una vez ubicada nuestra pieza en un ambiente concreto, pasaremos a analizar sus elementos:

El Disco. Compuesto de tres elementos fundamentales: el granate central, la filigrana de oro, y los triangulitos exteriores granulados.

Apenas tenemos paralelos de joyitas donde en un disco se presenten los tres o al menos dos de estos elementos juntos; sólo conocemos un pendiente del Museo de Nápoles (2) y el colgante de una gargantilla, éste con triángulos exteriores granulados, superficie abombada igualmente granulada y seis granates y una cornalina engastados en aquella. Procede de Nola, también cerca de Nápoles (3) (fig. 2). Lo más usual en joyitas con disco es que éste lleve una representación floral, a menudo una roseta, o bien una carita o cabecita femenina.

Por el contrario, la composición de nuestro disco es del todo similar a las de las plaquitas semicirculares o en creciente lunar frecuentes en el siglo III a. C. y que se presentan con el mismo tipo de colgantes. La única diferencia es que aquellos suelen llevar en la parte superior un entalle circular, oval o triangular con otra piedra además de la de la propia plaquita; también presenta en los extremos o puntas tres bolitas decrecientes superpuestas.

Presentamos a continuación cinco ejemplos característicos de joyitas con colgantes en creciente lunar que contienen los mismos elementos compositivos y en el mismo orden del disco de Cartagena:

a) Un par de pendientes del ajuar de una tumba etrusca de Bettona (Umbria). Actualmente en el Museo de Villa Giulia. Cronología aproximada: siglo III a. C. (4) (fig. 3).

b) Dos pares de pendientes en el Museo de Nápoles, de procedencia desconocida. Cronología estimada: siglos III-II a. C. (5) (fig. 4).

c) Un par de pendientes, procedentes de Cerdeña, hoy en el British Museum. Cronología estimada: siglo III a. C. (6) (fig. 5).

(1) Notas referentes al tipo general que presentamos, pueden hallarse en Higgins, R. A.: "Greek and Roman Yewelery", Handbooks of Archeology, London, 1961, págs. 154 ss.; Hoffmann, H. y Davison, P. F.: "Greek Gold. Jewelery from the age of Alexander", Mainz/Rhein, 1969, págs. 9 ss.; Becatti, G.: "Oreficierie antiche delle minoiche alle barbariche", págs. 87 ss., Roma, 1955.

(2) Siviero, R.: "Jewelery and amber of Italy. Collection in the National Museum of Naples", New York/London, 1959; p. 39, n. ${ }^{\circ} 118$, lám. 122a, también con anforita y cuatro colgantes. Siglo III a. C.; Breglia, L.: Catalogo delle oreficierie del Museo Nazionale di Napoli», Roma, 1941, págs. 44-47, n.os 124-125, lám. XX, 1.

(3) Hoffmann, H. y Davison, P. F.: op. cit., págs. 136-137, n. 48.

(4) Becatti, G.: op. cit., pág. 199, n. ${ }^{\circ}$ 408, lám. CVIII. Encima del creciente lunar: engaste circular con piedra perdida, flanqueado por dos volutas granuladas.

(5) Siviero, R.: op. cit., pág. 39, n.os 117-118, láms. 120b y 122a. Pueden verse también otros con plaquita semicircular o de disco en Breglia, L.: op. cit., págs. 44-47, n.os 121-146, pero sin fotografías.

(6) Marshall, F. H.: "Catalogue of the Jewelery Greek, Etruscan and Roman, in the departements of Antiquities, British Museum», London, 1969, pág. 278, n.os 2356-2357, lám. LI. 


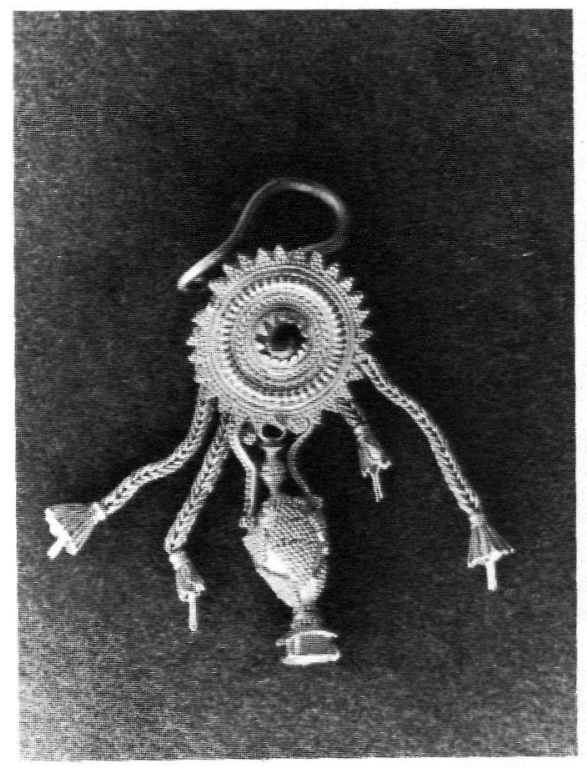

FIG. 1a

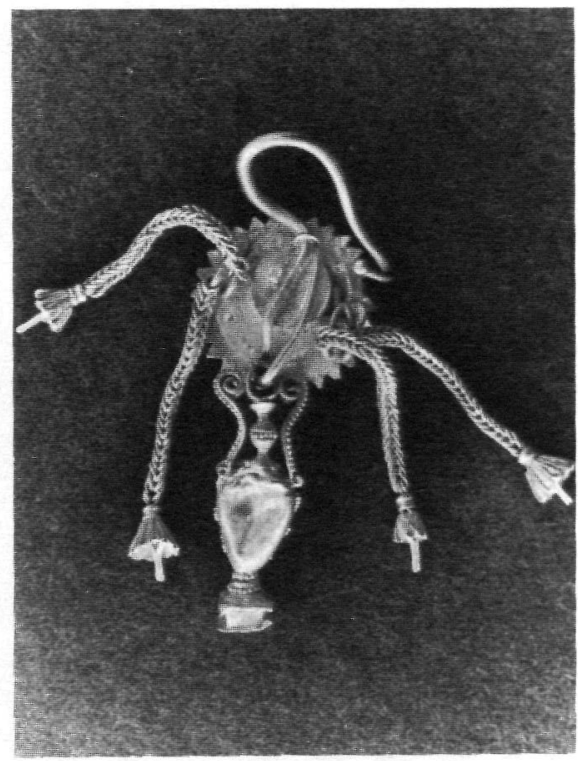

FIG. 1b

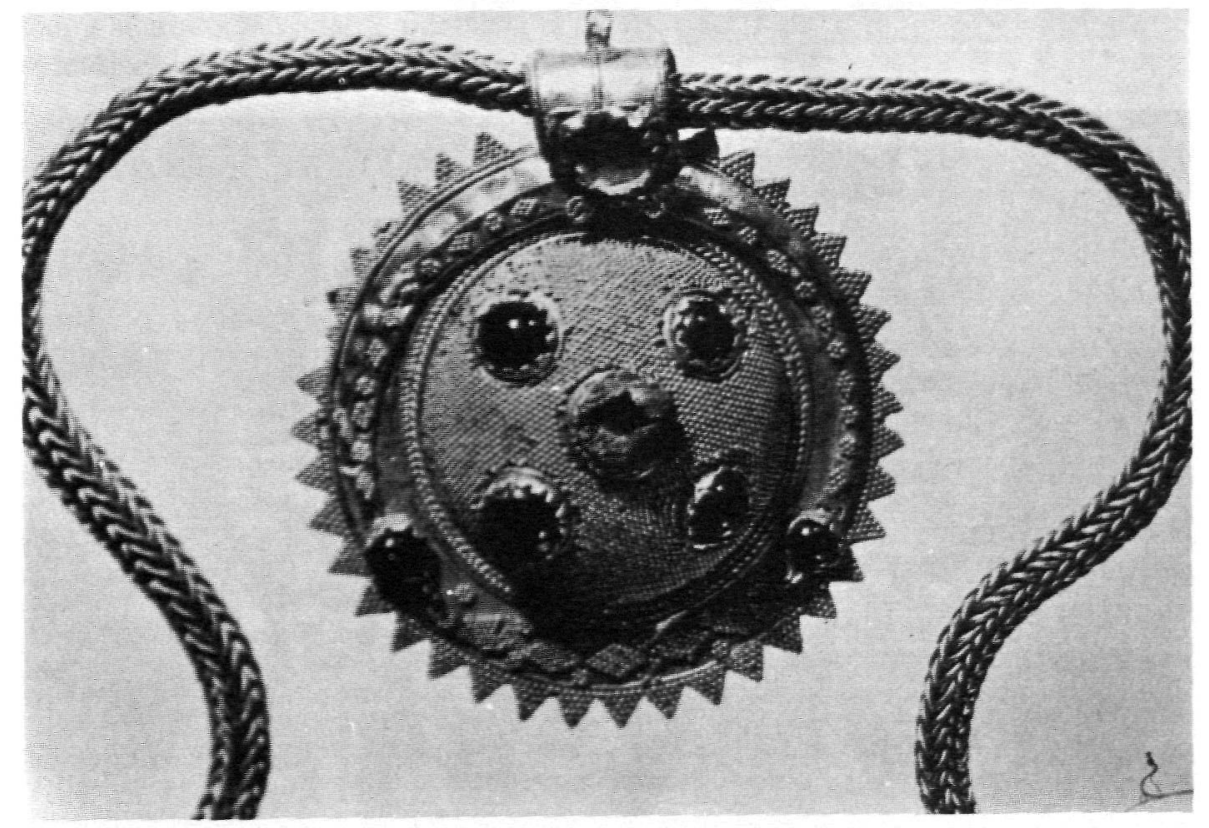

FIG. 2 


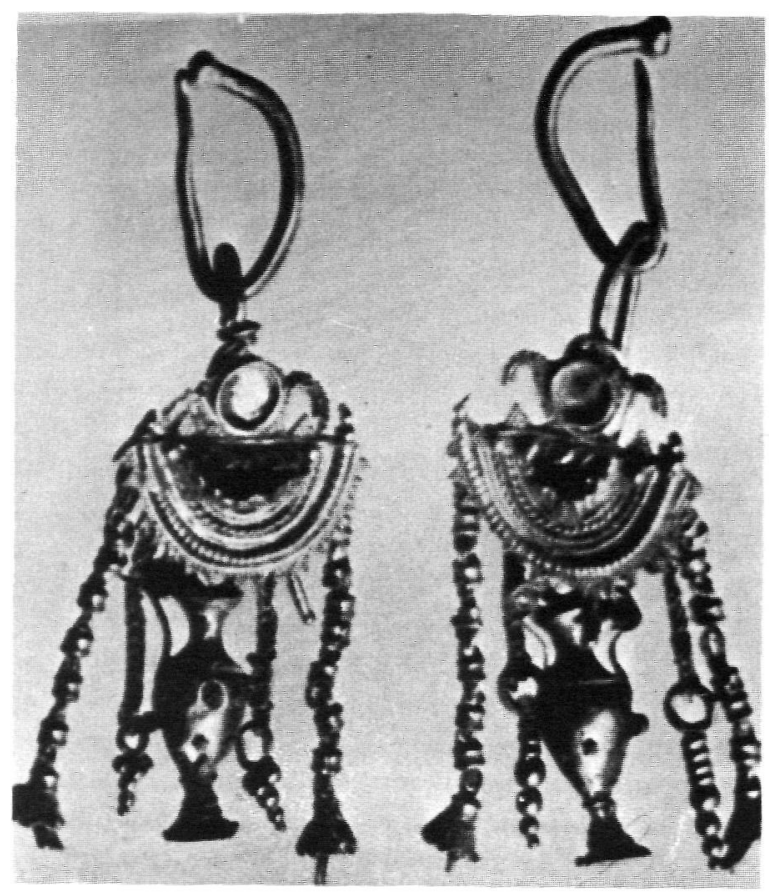

FIG. 3

FIG. 4

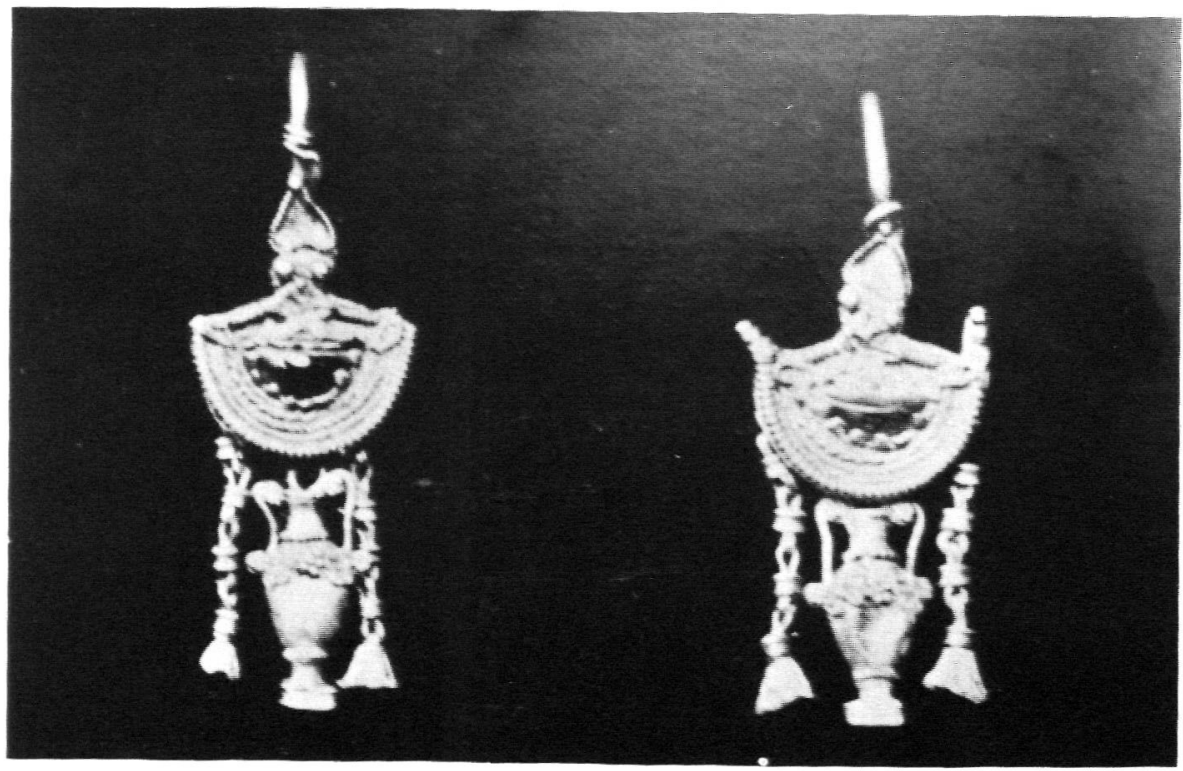


d) Un par de pendientes procedentes de Vulci, hoy en el British Museum. Cronología estimada: siglo III a. C. (7).

e) Un colgante de gargantilla o collar, procedente de Estanyol (Gerona). Cronología estimada: siglo III a. C. (8) (fig. 6).

Los tres primeros $(a, b, c)$ presentan además un colgante en forma de anforita con peana cuadrada similar a la nuestra, con decoración granulada en asas, cuerpo y a veces peana, y a los lados dos o cuatro colgantes de eslabones o trenzado, rematados por campánulas o bolitas.

Los pendientes del apartado d), con medio disco similar a los anteriores - si no idéntico-, y con otro granate flanqueado por delfines en la parte superior, tienen como colgante una figurita en pasta blanca y cuatro colgantes laterales a base de bolitas de oro y granates, acabados dos en campánulas y dos en bolitas decrecientes.

El colgante e) pende de una cadena o gargantilla de oro, hallada junto a otras joyas tesaurizadas dentro de una olla en un "yacimiento romano" en Estanyol (Gerona) a fines del siglo pasado (9). Lo interesante de esta pieza, que corresponde a una plaquita semicircular o en creciente lunar, con los mismos elementos decorativos - y dispuestos de la misma forma- que las plaquitas de los pendientes a), b), c), d), y el que aquí se estudia, es su presentación reutilizado como colgante de collar de lo que sería la parte fundamental de un pendiente: el disco semicircular que sostiene los colgantes. Aún puede verse en la parte cóncava del perímetro, el hueco dejado por los triángulos granulados donde iría seguramente otra piedra engastada como vemos en los aquí presentados a), c) y d). En el lugar donde el colgante se une a la cadena, pendería la figurita principal y los cuatro colgantes secundarios.

E1 anforita. Es el ánfora uno de los objetos más frecuentes utilizados como colgantes en joyitas helenísticas especialmente en pendientes de disco o plaquita semicircular (10), apareciendo ya en el siglo V a. C. y perdurando en época romana. $\mathrm{Su}$ "tipología» en lo que a los siglos III y II se refiere, no es muy compleja: se trata siempre de un ánfora esbelta de cuerpo piriforme, a veces algo grueso, alto cuello y boca pequeña, con dos largas asas que a menudo apoyan en voluta sobre el borde, sobresaliendo de éste. El pie suele consistir en una base plana formada por una peana cuadrada (como es el caso de la de Cartagena) o bien una base apuntada con varias bolitas decrecientes superpuestas. El cuerpo consta a veces de una piedra (granate, cornalina, cristal de roca), al cual la base y el cuello del ánfora hacen de entalle. Como puede deducirse, estas piezas suelen ser más recientes (fines del

(7) Becatti, G.: op. cit., pág. 199, n. ${ }^{\circ}$ 404, lám. CVII; Marshall, F. H.: op. cit., pág. 182, n. os 1677-1678, lám. XXXI. Según Marshall este par podría ser posterior en el tiempo al precedente, n. os 2356-2357. Otras piezas de procedencia itálica, con plaqueta idéntica a la citada: n. ${ }^{\circ} 1681$, lám. XXXI, n. ${ }^{\circ} 2359$, lám. LI.

(8) Serra Rafols, J. de C.: "Hellenistic Jewels at Estanyol», Fasti Archaeologici, IV, Annual Bull, of Class. Arch., (1951), págs. 417-418, epígrafe 4120, fig. 99; Becatti, G.: op. cit., pág. 107, n. ${ }^{\circ}$ 435, lám. CXIX.

(9) Ver nota 8.

(10) Becatti, G.: op. cit., pág. 88; Higgins, R. A.: op. cit., pág. 166; Marshall, F. H.: op. cit., colgantes con anforitas entre los n.os 2324 y 2371, lám. LI. 


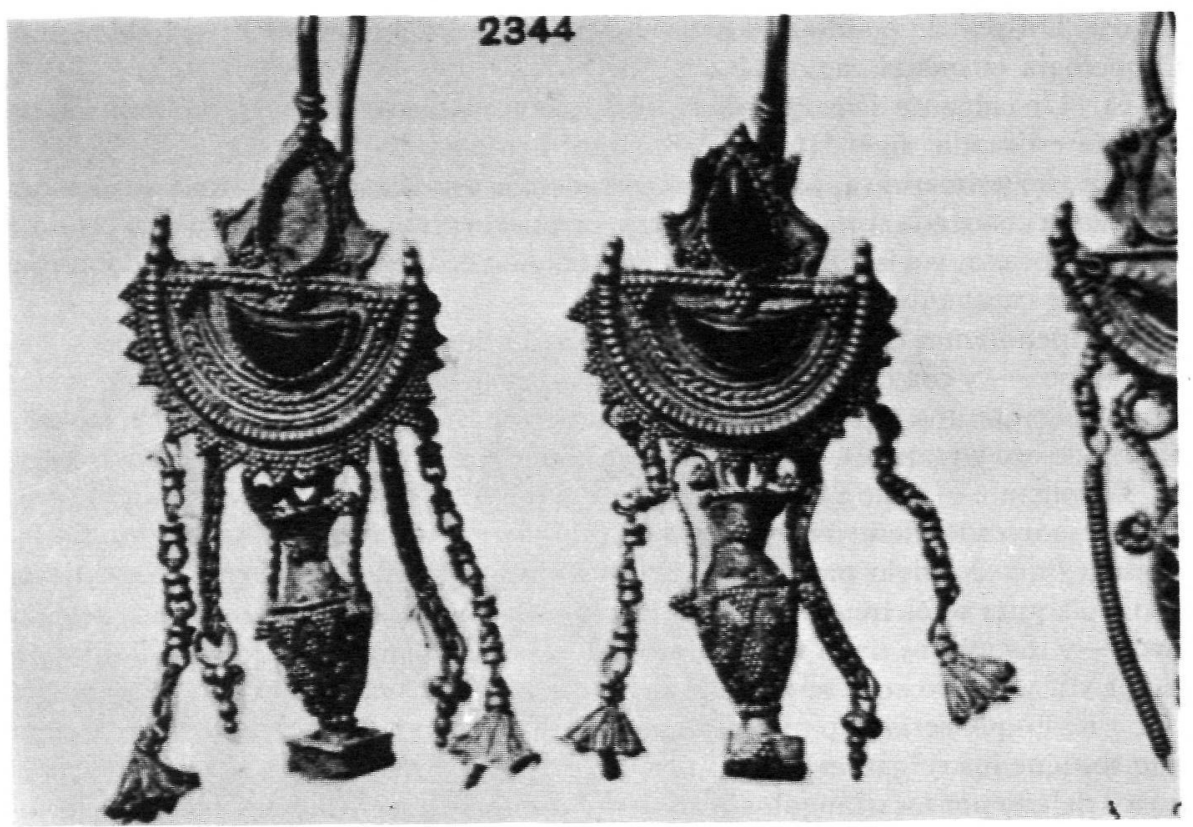

FIG. 5

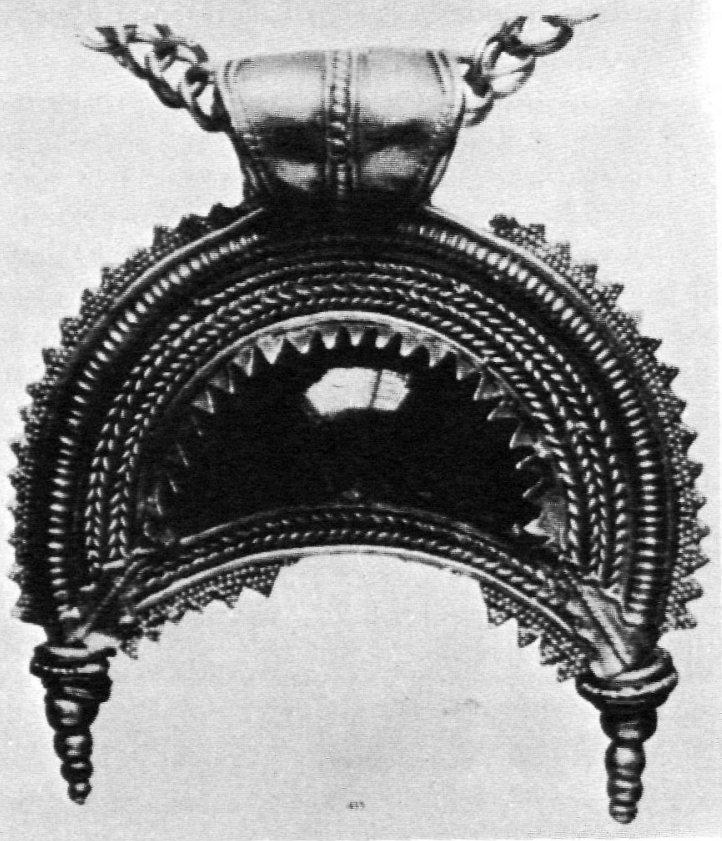

FIG. 6 
siglo III-II a. C.) que aquellas que tienen todo el cuerpo de oro y cuelgan a su vez de un disco o semicírculo afiligranado (11).

El granulado que a menudo decora estas anforitas formando hileras en las asas, o rosetas, triángulos, rombos, en el cuerpo y peana, es escaso en la orfebrería griega clásica, y es un motivo decorativo complementario en época helenística. Usado con profusión, es más típico de las costas del Mediterráneo Oriental en el siglo III a. C. (12), siendo frecuente hasta el siglo I a. C.; es sin embargo curiosa su presencia asimismo en piezas helenísticas de tumbas etruscas o en el sur de Italia, creemos que como piezas de importación.

Los colgantes secundarios. Formados como antes dijimos por cuatro cadenitas trenzadas de cuatro cabos acabadas en campánulas, son elementos de escasa significación identificadora; este trenzado lo vemos en joyitas ya desde el siglo $\mathrm{V}$ a. C., y en pendientes helenísticos es frecuente encontrarlo alternando con cadenetas de eslabones cilíndricos (13). Es habitual en joyitas de disco y otros colgantes del siglo III a. G., y si algo podríamos resaltar es la sencillez y elegancia que transmiten en nuestra pieza, sin colores o piedras variadas, entonando perfectamente con el disco.

Conclusiones. El pendiente de Cartagena es indudablemente helenístico, seguramente de fines de ese primer período que va del 330 al 200 a. C., conservando una armonía entre filigranas, granulado y piedras que se rompe después del 200 a. C. con la inclusión de más colores y presencia de mayores y variadas piedras.

A pesar de lo "normal» de todos los componentes, la configuración general del pendiente con la anforita, y detalles como el borde dentado del disco y la abundancia del granulado, nos acercan al Mediterráneo Oriental, donde estos detalles son característicos. Pero no podemos olvidar que la mayoría de los paralelos más cercanos, han sido hallados en Italia Central y Meridional, y bien pudo ser esa la procedencia de nuestra pieza de Cartagena. En cuanto a su hallazgo estratigráfico, la joyita apareció dentro de un paquete de material revuelto pero homogéneo, con cronología que oscila entre fines del siglo III y el cambio de Era. Nuestro pendiente pudo ser además objeto de atesoramiento o simplemente de conservación dentro de un mismo núcleo familiar, como igualmente pudo ocurrirle al de Estanyol.

(11) A estos efectos pueden compararse los pendientes n. os 2332-2333 del British Museum del siglo IV a. C. (Marshall, F. H.: op. cit., lám. LI), n. 408 de Bettona del siglo III a. C. (Becatti, G., lám. CVIII), y n. ${ }^{\circ} 48 \mathrm{~g}$, del siglo II a. C. (Higgins, R. A.: op. cit., pág. 166 y fig. 48).

(12) Veáse por ejemplo la pieza citada al hablar del disco, con cenefa de triángulos granulados (Hoffmann, H. y Davison, P. F.: op. cit., pág. 136, n. ${ }^{0} 48$ ). También los pendientes del British Museum de procedencia o estilo oriental (Marshall, F. H.: op. cit., lám. LI).

(13) Pueden encontrarse numerosos ejemplares en el British Museum: (Marshall, F. H.: op. cit., n. os 1653 a 1683; n.os 2330 a 2360); Hoffmann, H. y Davison, P. F.: op. cit., n. 20 en adelante; Higgins, R. A.: op. cit., págs. 160 ss. 